\title{
A Brief Tour into the History of Gravity: From Emocritus to Einstein
}

\author{
Panagiotis Papaspirou and Xenophon Moussas \\ Department of Physics, Section of Astrophysics, \\ Astronomy and Mechanics, University of Athens, Athens, Greece
}

\begin{abstract}
The History of Gravity encompasses many different versions of the idea of the Gravitational interaction, which starts already from the Presocratic Atomists, continues to the doctrines of the Platonic and Neoplatonic School and of the Aristotelian School, passes through the works of John Philoponus and John Bouridan and reaches the visions of Johannes Kepler and Galileo Galilei. Then, the major breakthrough in the Theory of Motion and the Theory of Gravity takes place within the realm of Isaac Newton's most famous Principia and of the work of Gottfried Leibniz, continues with the contributions of the Postnewtonians, such as Leonhard Euler, reaches the epoch of its modern formulation by Ernst Mach and other Giants of Physics and Philosophy of this epoch, enriches its structure within the work of Henry Poincare and finally culminates within the work of Albert Einstein, with the formulation of the Theory of Special Relativity and of General Relativity at the begin of the 20th century. The evolution of the Theory of General Relativity still continues up to our times, is rich in forms it takes and full of ideas of theoretical strength. Many fundamental concepts of the Epistemology and the History of Physics appear in the study of the Theory of Gravity, such as the notions of Space, of Time, of Motion, of Mass, in its Inertial, Active Gravitational and Passive Gravitational form, of the Inertial system of reference, of the Force, of the Field, of the Riemannian Geometry and of the Field Equations. These primary fundamental theoretical and structural notions appearing each time in the corresponding Theories of Gravity and within the various Paradigms of the Gravitational interaction. We shall refer briefly to the History of Gravity, mentioning only a few landmarks or great personalities which shaped these fundamental physical and epistemological notions.
\end{abstract}

Keywords: Kinematics of Bodies, Dynamics of Bodies, Space, Time, Mass, Gravitational Force

\section{INTRODUCTION}

The list of important Philosophers, Scholars and Scientists who contributed to the formulation and the unraveling of the notion of the Gravitational interaction include the Atomists, such as Democritus, the Platonic and Neoplatonic doctrines, the Physics of Aristotle and then, passing on to the Medieval Period, the works of John Philoponus, Nicholas of Cusa and John Bouridan, the ideas of Johannes Kepler and of Galileo Galilei, continues to the publication of the famous Principia of Isaac Newton, develops further within the works of Euler and Lagrange, Mach and Poincare and reaches its peak Corresponding Author: Panagiotis Papaspirou, Department University of Athens, Athens, Greece within the formulation of Einstein's Theory of General Relativity, in its first proposed form.

We shall start our conceptual tour in the History of Gravity from the very early insights of the Atomists and end up to the first phases of the Theory of General Relativity, mentioning only a few of the beautiful landscapes we encounter.

This route begins with the World-view of an Atomistic Universe, continues with the works of Aristotle, which were been regarded as the exemplar for the Sciences of Mechanics, Astronomy and Cosmology and the Neoplatonic notions of mass and force, the theory of the Impetus by John Philoponus, takes a 
completely new turn within the framework of Isaac Newton's Principia and the further development of the Newtonian heritage within the works of Euler and Lagrange and other, but also within the modernization of the concept of Inertia and of Relativirty within the thought of Ernst Mach and its eponymous principle and Henri Poincare, the first to introduce elements of the Scientific discipline of Chaos within Celestial Mechanics. Finally, we close this historical journey with the formulation of Einstein's Special and General Theory of Relativity, grounded on the rich mathematical structure of Bernhard Riemann's Differential Geometry, a new and contemporary form for structuring and interpreting the fundamental interaction of Gravity.

\section{THE HELLENIC TRADITION OF THE THEORY OF GRAVITY}

\subsection{Introduction}

The Hellenic Tradition, especially with the advent of the Ionian Renaissance of the 6th century, introduces for the first time the realm of Philosophy and Science, as we understand them in our epoch and the Science of Physics in particular, both its theoretical, as well as its experimental branch, an undertaking that reaches its peak within the Hellenistic and Alexandrian tradition (Boschiero, 2010). The whole series of the fundamental metaphysical questions are posed and the relevant philosophical and scientific issues become the subject of intense inquiry of every Philosophical School belonging to this tradition.

Within this Tradition three most fundamental Worldviews and attitudes, manners of Thought towards Nature, are born and developed within the historical course of History. The first fundamental approach towards the totality of the physical phenomena and especially for a primal form of the gravitational interaction, is the idea about the Atomic structure of the Universe. The second deep fundamental idea refers to the Creator, the Demiourge of the mathematically ordered Universe, the Cosmos and his role as a skilfull Mathematician. The third fundamental approach is the experimental investigation of the physical phenomena, which reaches its peak within the Hellenistic and Alexandrian Tradition and especially within the framework set up by the Giants of Science in the cosmopolitan city of Alexandria, up to the death of Hypatia (Cornford, 1922; Russo and Levy, 2004).

\subsection{The School of the Atomists: The Metaphysics and Physics of Democritus}

Democritus of Abdera can be considered as one of the most influential Philosophers of all times and one of the founders of the Atomistic School in Philosophy. The atomistic conception about the nature of Matter, the various kinds of Atoms moving through the void and the justification of all physical phenomena on the basis of the interactions among the Atoms continued their influence at least up to the Post-newtonian age of Physics and especially of Classical Mechanics and contributed to the birth of Statistical Mechanics, through the pioneering work of Ludwig Boltzmann. Lucretius, in his famous work De Rerum Natura, offers us perhaps the most famous and popular exposition of the Atomistic Philosophy, Metaphysics and Physics.

Lucretius informs us that the causal and spatiotemporal order of the physical phenomena are deduced by the nature of the atoms and their collisions within the void.

The Principle of the Permanence of Matter, as well as the Principle of Indestructibility of Matter, which belong to the pillars of Democritus' Metaphysics, are stated at the begin of this study. The phenomenal world of appearances and transitory entities is characterized of secondary qualities, the primary physical qualities stem from the size, movement and shape of the colliding atoms, generating ever-changing configurations and combinations. Space is conceived as an infinite physical substratum, an extension which remains unaffected by the materialprocesses, while time is another physical extension, which flows continuously, during the evolution of the Democritian Universe. Space contains the void, the atoms and all the collisions between them, which can be regarded as contact collisions or as collisions stemming from a kind, a precursor of the Force of Gravity. Space, in its atomistic definition, is regarded to be an infinite, isotropic and homogeneous receptacle of the physical bodies, an idea, although altered and refined, reoccurring within the Newtonian Paradigm, especially in the definitions of Space and Time (Jammer, 1954).

The atomic structure of matter introduces operational definitions and interpretations about the concept of Mass, although the Mass as Quantitas materiae, or as Dynamical Mass, that is in its inertial or its two-fold gravitational notion is not discussed in detail (Jammer, 1997).

Among the indirect influences of the Theory of Gravity we can mention two characteristic examples. 
Giordano Bruno, in his treatise "On the Infinite Universe and Worlds" revives the Democritian and Epicurean Philosophy of Atomism and describes the World- Picture of an infinite plenum contained within an infinite void. This is an example which belongs to the Cosmological Doctrines of the Atomistic School and which influenced indirectly some the cosmological Paradigms that followed, Paradigms which heavily relied on the novel Newtonian Paradigm.

As for another face of the importance of the Atomistic School we can mention that with the new advent of the Atomistic doctrine, the Scientific and Philosophical branch of Alchemy downfalls, while the modern quantitative Science of Chemistry is newly born. Newton himself considered a plethora of natural forces, divided into the broad categories of the contact forces and the forces that act at a distance, besides the Force of Gravity and tried to incorporate them fully within his scientific framework. This indirect influence upon Newton's achievements is also worth-mentioning (Ducheyne, 2007).

The Atomistic School already includes its own particular descriptions of Space, Time, Motion and Force, also incorporating a precursory scheme of a Theory of Gravity.

\subsection{The Neoplatonic Tradition and its Views about Space, Time, Matter and Force}

The concept of substance and of interaction plays also a fundamental role within the Neoplatonic delta of the philosophical traditions. The ordered Universe, obeying mathematical Principles of Symmetry, Analogy, Symphony and proportion, which serve as underlying fundamental Physical Principles as well, has to contain invariant, eternal and absolutely conserved entities and quantities, which refer to the very same idea and characteristics of the Demiourge of Cosmos (Cornford, 1922; Burnet, 1914; Jammer, 1954). Beginning with Plato's doctrines and continuing with its Neoplatonic successors, Nature is purely geometrized (Jacob, 1991).

Space is considered as permanent, as the overall matrix, where all physical phenomena take place, while within the Neoplatonic understanding of Cosmos, the physical bodies are identified with concrete geometric forms, according to the doctrine and Paradigm of the five convex regular polyhedra, the five Platonic solids. These solids interact with each other, or transmute to each other in certain analogies, their polygonal faces being composed by two certain kinds of right triangles.
The concept of Mass, according to the Neoplatonic doctrine, is conceived as being absolutely passive and inactive, therefore there does not exist an operational way in order to attribute to it a measurable physical quantity (Jammer, 1997).

One of the most influential ideas of the Neoplatonic tradition refers not only to the concept of mass, although not distinguished into its inertial an gravitational notion, but also to the concept of Force, which as an Idea of the Demiourge acts on the passive matter, or materia, with these kind of forces completing the whole scenery of all physical processes of Motion and Tranformation within an organically conceived Cosmos (Jammer, 1957). This notion shall travel through historical time and successive generations of scholars and polymaths of the Natural Sciences and finally reach the epoch of Johannes Kepler and Isaac Newton, through the exact definition and introduction of the Gravitational force.

\subsection{The Legacy of Archimedes}

Archimedes, also, must have been close to the formulation of the concept of mass, as we understand it in its modern terms, in his famous treatises of Statics and Hydrostatics. Archimedes defines the center of mass of the bodies, formulates for the first time in the History of Mechanics the law of the levers in Statics and comes close to some modern conceptions about the nature of the physical forces and the gravitational force, as well. In his works we can readily encounter the forerunners of the notions of specific gravity and of density, although they are never mentioned explicitly (Nowacki, 2010; Jammer, 1997).

Archimedes further develops a heuristic method of for the determination of areas and volumes of complex nature by "weighing" them. We can clearly follow his approach in his famous treatise "On the Quadrature of the Parabola", where he applies extensively his heuristic mathematical arguments and then proceeds with the strict mathematical proof of the obtained theorems (Spandagos, 2011).

Archimedes belongs to the Scientific spirit of the Hellenistic and Alexandrian epoch and his idiosyncratic way of perceiving the physical reality does not always confirm either with the Platonic, nor the Aristotelian School (Russo and Levy, 2004). Archimedes, belonging to and at the same time shaping this tradition, can be regarded as the father of the complicated Planetaria and the Computing devices which reproduced the celestial motions, with the most famous example being the Antikythera Mechanism (Moussas et al., 2007; Moussas, 
2012; Henriksson, 2009). His legacy continues to the Byzantine, the Arabic and Islamic and the European Civilization, where he is regarded to belong to the most influential and greatest Mathematicians and Physicists of all times.

\section{THE MEDIEVAL APPROACH ABOUT SPACE, TIME, MOTION and GRAVITY}

\subsection{Introduction}

The Aristotelian Theory dominates within the curricula of all the European Universities and becomes the exemplar for the Theological, Metaphysical and Epistemological approach towards the physical reality, thus addressing the problems of Space, Time, Motion and Change within Nature. The Substance of the material bodies, the Change within the physical phenomena, the Interaction of the various elements of Cosmos, its Structure, the Creation of Forms within it, the Dynamical realization of the physical processes, these constitute major problems within the thematics of the Aristotelian doctrine.

Space, within the framework of the Aristotelian doctrine, belongs to the category of the continuous quantities, while Time exhibits the same attribute. Space is furthermore conceived as the sum of all places occupied by a physical body, since the existence of the void space is both logically and physically unacceptable. Space is the carrier of the qualitative differences we encounter in Aristotle's Theory of the Elements of Nature and in the Aristotelian Cosmological Paradigm, "saved by" the Ptolemaic Astronomical Model (Jammer, 1954). These elements and their motions, constitute a dynamical field structure and space is the substratum where all the "natural motions" of the elements, both in the sublunar region of the Universe, as well as in the region of the celestial bodies, take place.

Also, according to the Aristotelian view, volume and therefore weight, cannot play the role of a primary quantity of matter, since, just to raise a Peripatetic argument, the matter of a physical body may remain identical during the transformation of its volume, just as in the process where water is transformed to air. The matter, within the Aristotelian conception, is perceived as being organic in nature, there does not exist any permanent or invariant quantity which would serve for its description or identification and is always passive, acted upon by external forces, thus it does not possess Inertia (Jammer, 1997).

Perhaps the only exception within the Medieval period, the highly idiosyncratic Neoplatonic Philosopher and Polymath Nicholas of Cusa, who introduces in his works the weighing of the mass as the proper reliable and accurate method of research, in general and for the case of determing the properties of mass in particular, especially in his work De Staticis Experimentis (Jammer, 1997). It is not a coincidence that Nicholas of Cusa, as a Mystic, a Theologician and a Physical Philosopher influences greatly the thought and work of Johannes Kepler, who also proposed elaborate insights about the notion of the Gravitational force during his research into the planetary motions.

\subsection{John Philoponus: The Theory of the Impetus}

John Philoponus introduces for the first time, in Medieval Philosophy, the most important concept of impetus and can be regarded as the forerunner of Galileo's notion of inertial mass. His theory regards both the concept of mass and the concept of motion through space and within the passage of time, therefore it can be regarded as related with the Theory of Gravity, in which framework these problems have to be precisely formulated and adequately solved. The theory of impetus is a decisive step towards the Keplerian, Galilean and Newtonian Metaphysics and Physics of motion, departing strongly from the Aristotelian doctrine (Elazar, 2011).

According to Philoponus' Theory of Impetus, the projectile motion has to be considered as caused by a kinetic force impressed on the material body by the mover and exhausts itself during the course the movement takes place. This force, in its Neoplatonic description, is incorporeal and is called as the "energy", (energeia, in Greek), a sort of activity also attributed to the nature of Light, in Philoponus' Theory of Light.

John Philoponus stands as a landmark in the Neoplatonic tradition, although his influence had to be performed under the casting of the shadow of the anathema attributed to him because of his idiosyncratic notion of the Trinitarian existence of God. His legacy continued within the Medieval European Tradition in the form of the work of John Buridan, another outstanding Figure who also influenced greatly all the forthcoming investigating minds in the historical flow of time (Kalachanis, 2011).

\section{JOHANNES KEPLER: HIS APPROACH TOWARDS GRAVITY}

Kepler is convinced that the Copernican heliocentric model of the Universe corresponds in an absolute and definite manner to the physical reality and enriches the Copernican Paradigm with the statement of his 
eponymous three Laws of planetary motion, an ingenious labor which served as one of the key elements for Newton to derive his own Theory of Gravity (Theodossiou et al., 2002). Kepler, in his attempt, combines the Christian Neoplatonic elements of a Geometrized Universe with his novel approach of a Dynamical Cosmos driven by specific Laws of Nature.

At the historical epoch of Johannes Kepler the prevailing Paradigm about the motions of the celestial bodies within the Science of Astronomy considered the perfect cyclic motion as the modulator of these celestial motions, because of the geometrical simplicity, the aesthetic perfection and the continuity of the circle. The celestial motions were considered to be generated by combinations of perfect circular motions, that is by the corresponding systems of deferent circles and epicycles. Nicolaus Copernicus believed strongly in the necessity of this epistemological doctrine and was admired by his cotemporaries supporting the Heliocentric Universe because within the framework of his theory all planetary motions were reproduced by circles and epicycles, abandoning completely the usage of the notion of the equant. The notion of the equant was considered to be highly problematic in the Ptolemaic Geocentric and Geostatic Cosmological Paradigm and many attempts for its successful replacement were attempted during its long history of evolution and transformation.

Kepler is faced with a serious problem of theoretical origin, that is whether the elliptical paths of the planets, that is according to the statement of his first Law of planetary motion, do correspond to a natural motion dictated by a Physical Principle or a Physical Law (Dreyer, 2011). His Universe is considered as being dynamical, therefore he searches for reducing the geometrical, kinematical and dynamical characteristics of the planetary orbits to a deeper underlying causal relation, a form of a Physical Law that has to be introduced within the realm of Astronomy, surpassing the old and inadequate astronomical tradition.

He comes to the idea of the existence of a physical force, of an anima motrix, which is responsible to keep the planets on their motion around the Sun, the physical center of the Universe and the symbol of the Ideas of the Demiourge of Cosmos, as stated within the Neoplatonic and Neopythagorean doctrines, World-views and manners of thinking that influenced greatly his own Thought (Papaspirou, 2012). Kepler, in his work, introduces the concept of mass, corresponding to the perception of matter and the concept of force, corresponding to the motory intelligence which keeps the masses on their continuous motion around the Sun, the true generator of this motion. Kepler is also heavily influenced by the work of his contemporary William Gilbert, the famous at his age De Magnete, among all the other influential works of this original thinker, the first complete modern description of the magnetic force in the European Civilization and considers the nature of the gravitational force as being similar to the magnetic, a force that stems in the form of imaginary lines out of the surface of the center of the Universe, the Sun.

Thus, he approaches under a modern light the problem of the formulation of a gravitational force and although he does not come into a precise formulation of the Law of gravitational attraction, his work becomes one of the pillars of Isaac Newton's Theory of Gravity.

\section{GALILEO GALILEI: HIS APPROACH TOWARDS KINEMATICS}

Galileo considers the primary qualities of Matter, that is the geometrical qualities which encompass the notion of the shape, the size, the location and the contiguity, the arithmetic quality of number and the kinematic quality of motion, while within his thought the quality of mass is being considered as a primum.

Galileo, in his work about the Kinematics of the physical bodies, introduces the concept of the inertial frame of reference and the Galilean Principle of Relativity (Ferraro, 2007). In modern terms Galileo's Principle of Relativity can be defined as: If there exists an inertial frame of reference, then there exists an infinity of inertial frames, obtained one from another by a rectilinear and uniform motion of translation. Galileo mainly contributes to the Kinematic aspect of the motion of the physical bodies, whose motion is mainly originated under the influence of a constant force, while according to his view, we ought reject any theoretical conjecture about the true essence of the term "force". Both Kepler, as well as Galileo, stand with their great achievements as complementary Figures, whose spermatic Ideas and insights contributed greatly to the birth of the Newtonian Theory of Gravity.

\section{ISAAC NEWTON: THE PHILOSOPHIAE NATURALIS PRINCIPIA MATHEMATICA}

\subsection{Introduction}

Newton publishes his famous Mathematical Principles of Natural Philosophy for the first time in 
1687, where he introduces the kinematical and dynamical description of the physical phenomena regarding the motions and the interactions of the physical bodies, unifying the physical reality between the celestial and the earthly realm and more specifically, by introducing his famous law of Gravitational interaction. This most famous work in the History of Physics serves at the same time as a fountain of ideas, as the birth of a Theory in Physics and as the exemplar of the Newtonian Paradigm in Physics, the Natural Sciences and the Social Sciences, as well (Jammer, 1954; 1957; 1997).

The deep and influential thought and especially the work of Johannes Kepler, which includes the formulation of his three eponymous Laws of planetary motion, that is the Law of the Ellipses, the Area Law and the Harmonic Law, serves as a productive basis for Newton, who finally succeeds to synthesize them within the framework of his Theory of Gravity. Newton formulates his Three Axioms of Motion, while within the formulation of the Universal Law of Gravitation among the material bodies, Newton is able to reproduce all of Kepler's three laws and unify the celestial and earthly phenomena. Newton's Theory of Gravity ended all opposition to the work of Johannes Kepler, that is to his three Laws of planetary motion (Wilson, 1970).

Newton considers time as a physical primary notion, which flows independently of the physical systems and is regular. He also considers space as independent, as a substratum which underlies the physical reality and acts on the physical bodies, but cannot be acted on. Newton distinguishes between the absolute, true and mathematical quantities and their apparent, relative and common notions. This work reformulates the concepts of Space, of Time, of Motion and of Mass, in its inertial and gravitational nature and paves the way towards the modern physical description of reality (Ducheyne, 2007). The philosophic importance of Newton's work surely exceeds the limits of Mechanics, since it is considered by the forthcoming generations of scientists and scholars to be the exemplar of any scientific theory, in any scientific field, which aims to describe the physical reality (Kuhn, 1996).

Newton also follows, in some respect, the doctrine of substantivalism, where space and time are considered to be real entities, necessitated by God, the Demiourge of Cosmos, within its eternal and omnipresent existence. He also introduces the concept of force, in its modern usage, for describing the Gravitational interaction, distinguishes among many types of physical forces, conducts experiments in order to reveal their true nature and finally addresses the problems related with the gravitational interaction among the physical bodies (Jammer, 1957; Westfall, 1973).

The essence of the Father of the Theory of Gravitation can be found in Newton's General Scholium, where he expands his own views about central scientific issues, of varied nature, such as the epistemological, the methodological, the experimental issues, as well as the metaphysical and theological issues, which constitute a great synthesis of all these elements within Newton's conception about Nature (Ducheyne, 2007). This is not uncommon for all the Spiritual Giants who flourished at those particular ages and as a first example we can mention the case of Johannes Kepler. Newton asserts in the General Scholium that there exists ancient knowledge supporting the truth of four basic propositions for the justification of his own views about space, time, matter and the gravitational force: that matter attributes an atomic structure, that matter moves through the void under the influence of the gravitational force, that the gravitational force offers a unifying and universal physical principle, applicable throughout space and time, that the gravitational force varies as the inverse square of the distance between two bodies and that the cause of the gravitational force refers to the direct action of the Creator of the Universe, as the very first Cause within a hierarchically structure of causes, while he is unable to determine the Cause of the force of Gravity.

Newton is fully aware of the conceptual problems raised within the formulation of his Theory of Motion and Gravity, especially the problem of defining the Inertia of a physical body and the problem of the "action at a distance", problems which shall find their answer only with the advent of the epoch, between the ends of the 19th century and the beginnings of the 20th century.

\subsection{Newton's Concepts about Space, Time, Force and Mass}

According to the World-view of Newton, Space, Time, Mass and Force correspond to real, objective physical entities and as the most essential attributes of the physical reality, whereas Geometry is not considered to be as abstract, but a branch of the Science of Mechanics (Jammer, 1954; 1957; 1997). Newton encompasses Space as absolute in its own nature, without relation to anything external, always similar and immovable, while the relative space is considered to be as movable dimension or measure of the absolute space. The concept of Absolute Time, also as considered by 
Newton, relates to a continuous, absolute and everflowing physical substratum, while the combination of Absolute Space and Absolute Time built the framework, constitute the "theatrical scene" where all collisions of physical bodies take place, generated by contact or by gravitational forces, a framework that acts, but cannot be acted upon. According to Newton, Absolute Space is being introduced as the correlate of Mass, following the steps of the Atomists who regarded the void as the correlate to the atoms (Ducheyne, 2007; Jammer, 1997). The absolute nature of Space and Time, within Motion takes place, is intensely correlated with the Absolute and Eternal existence of God.

Isaac Newton introduces the concept of the Physical Force, of course not for the first time, but in terms which stand more closely to our modern conception of a fundamental interaction, in terms which is are being developed further by the Post-newtonian School of Thought and which acquire their perfection at the end of the $19^{\text {th }}$ century, that is at the end of the Mechanistic Worldview. Newton, as well as Kepler exhibit an Ianuslike approach towards the physical reality, always being impressed and influenced by the Medieval and Renaissance ways of perceiving the physical reality, while at the same time introducing breakthroughs and cataclysmic Revolutions in the Science of Astronomy, of Mechanics and of the Physical Sciences. Especially, Newton's Principia serves as the exemplar of the overall scientific practice for centuries and is admired as such.

In particular, Newton introduces the Universal Law of Gravitational interaction axiomatically, thus the concept of the Force of Gravity and then proves mathematically and by physical argumentation, that all the up to his times acquired Astronomical knowledge confirms with this law, especially and more significantly with Kepler's laws of motion of the celestial bodies (Wilson, 1970). The Universal Law of Gravitation shall prove to be one of the most influential exemplars for the forthcoming Physical Theories, for example in Coulomb's formulation of his eponymous Law.

Newton is fully aware of the conceptual shortcomings of his Theory, for example he finds the idea of "action at a distance" meaningless and unphysical, or even as an unsolved issue the identification of the three notion of Mass, that is the Inertial, the Active and the Passive Gravitational Mass. On the other hand, the triumphal success of his theory on an experimental level, for correctly reproducing the kinematic and dynamical characteristics of the moving bodies, placed for many generations these issues close to the fringe of the Newtonian Paradigm.

This Paradigm underwent many transformations, during its evolution, by the works of Euler, Lagrange, or even by the contribution of Ludwig Lange, who introduced for the first time an elaborate notion about the Inertial System of Reference, which is not stated explicitly within the Principia. We mention these facts for demonstrating that the famous treatise of Newton differs in some respects from the Axiomatic Formulations of Classical Mechanics that followed by the forthcoming generations of Physicists (Arnold, 1989), while the Creator of Classical Mechanics and the Theory of Classical Gravity recognized these aspects from the very begin (Ducheyne, 2007; Suisky, 2008).

\subsection{The Philosophical and Scientific Contribution of Gottfried Leibniz}

Descartes, as well as Gottfried Leibniz, two of the Giants of the Cultural Heritage, also contribute to the formulation and interpretation of a Theory of Gravity, with Leibniz playing an important role, since his insights and ideas seem to be very close with our modern understanding of the gravitational form.

According to Leibniz, the Newtonian Paradigm of the gravitational interaction is confronted with the serious epistemological problem of the identification of the three heterogeneous categories of mass, that is the inertial mass, the active gravitational mass and the passive gravitational mass. The Leibnizian understanding of the concept of mass relies heavily on his Doctrine of Monads, where he also distinguishes between primary and secondary properties of matter and where he also introduces the notion of the interaction between the material particles, which comprise the physical bodies and their properties. Leibniz observes reality as generated mainly by mutual effects and interactions among the physical bodies, which are also identified with the observable and measurable ones.

Moreover, Leibniz heavily objects the notion of the Absolute nature of Space and Time, he considers as a physically relevant kind of motion the relative motion among the material bodies and as not meaningful their motion with respect to the inertial frame of reference of the absolute Space and Time (Bouquiaux, 2008). Leibniz claims that the physical observation between two particles, staying free upon the action of any force and moving with constant velocity with respect to absolute Space, cannot reveal which of the particles is at absolute rest and which is not. Newton is fully aware of this 
concrete conceptual difficulty within his own system and proposes the famous rotating vessel experiment, where he uses elaborately dynamical and not kinematical methods for determining which bodies undergo an inertial motion and which bodies undergo a relative acceleration (Ferraro, 2007; Dicke, 2011; Narlikar, 2011). Leibniz introduces the "equipollence of hypotheses", he articulates a general equivalence principle, where in any system of interacting bodies any hypothesis that a particular body is at rest is equivalent to any other body comprising this system.

The Leibnizian Doctrines of Monadology and Dynamism still continued to influence important Thinkers and contributors to the Science of Mechanics, even if their philosophical presuppositions remained at the fringes of the normal conduction of scientific activity within the Newtonian Paradigm.

\section{LEONHARD EULER: THE AXIOMATIZATION OF CLASSICAL MECHANICS}

Many Giants of Classical Mechanics and Physics in general, have contributed to the furthermore development of the Newtonian Theory of Gravity and the establishment of a novel conceptual basis for the framework Newton proposed, defined and applied successfully, such as the Figure of Lagrange. Among these great Figures, we can mention briefly the case of Leonhard Euler, one of the most influential Mathematicians and Physicists of all times, who contributed as one of the first of Newton's successors, to this direction.

Euler attempts to construct all the structure of the Mechanical Science, the Science of Newtonian Mechanics, within a system of Axioms, Definitions and Postulates, thus demonstrating the Apodictic character of Newton's Law of Gravity and of his three Axioms of Motion (Suisky, 2008).

Euler formulates in a more general fashion the totality of the Newtonian Laws in the form of differential equations relating the concepts of motion, force and torque, including the deformable bodies. Thus, Newton introduces for the very first time and Euler generalizes. Among Euler's great achievements, we can mention the following: Euler, in his first Treatise on Mechanics, titled as Mechanica, sive motus scientia analytice exposita, introduces the exact concept of the point mass, studies the acceleration as a kinematical quantity of motion which can be defined at any curve the material particle travels and further use the concept of the vector to describe the velocity, the acceleration and other kinematical quantities of the moving particle, extending the use of this geometrical object from Statics into Dynamics. More importantly, he also develops the first equations of motion for a rigid body, which, as an axiom, include all Principles of Mechanics (Jammer, 1957). Euler axiomatizes the concepts of Absolute Space and Absolute Time, while the notion of Mass, as the carrier of vis inertiae according to Newton, is re-introduced, according to Euler, as a numerical coefficient. It is only that within the framework of all Post-newtonian Giants of Classical Mechanics, the triple nature of Mass, that is the inertial mass, the active gravitational mass and the passive gravitational mass, are considered de facto as equal. This epistemological and methodological problem within the Newtonian Paradigm finds its resolution only within Einstein's Theory of General Relativity.

\section{THE MODERN CONCEPTS ABOUT GRAVITY}

\subsection{Introduction}

At the end of the 19th century a quantitative, operational determination of the concept of mass, of the gravitational interaction and of the concept of the field is being introduced by various great Physicists and Philosophers of Science. The concept of the field, which has also a long history of development, arises most naturally within Maxwell's Theory of Electromagnetism. The Electromagnetic field replaces the idea of the Electromagnetic force, because of, among other issues, the finite velocity of the propagation of light in vacuum. Thus, the idea of the Force is being abandoned, while the medium of the propagation of the interaction, the Electromagnetic or the Gravitational, plays the role of the primary physical concept and becomes the main object for theoretical investigation and for the establishing of the Modern Theories of Physics. The concept of the field shall acquire a very deep significance and a most prominent place within the Theory of General Relativity (Vizgin, 2011).

\subsection{The Work and Influence of Ernst Mach}

Ernst Mach, through his philosophical contribution, in general and his reformulation of Classical Mechanics, stands as a landmark in the History of Gravity, even indirectly, with the formulation of his eponymous Principle and the influence he exerted upon Albert Einstein for the formulation of the Theory of Special Relativity (Dicke, 2011; Narlikar, 2011). 
Ernst Mach objects to the concept of Newtonian space, as a physical underlying substratum which acts on the physical bodies, but cannot be acted upon. Mach, driven by his strong anti-metaphysical oppositions, tries to eliminate the "conceptual monstrosity of absolute space" out of the Science of Mechanics and replace it with his own functional definition of the Inertia of the physical bodies. According to his view, the unaccelerated motion of a material particle does not any more refer to Absolute Space, but to the center of mass of all the masses in the Universe. Newton's agents of God's supervenience are replaced by purely mechanical interactions among the material bodies. He also criticizes heavily the famous "basket experiment" of Newton, finding the interpretation Newton gave himself as inadequate and physically meaningless. Ernst Mach belongs to the Positivistic movement of his age, while his eponymous Principle will be incorporated as a major conceptual factor both within the Theory of Special Relativity and within the Theory of General Relativity (Jammer, 1954).

\subsection{Bernhard Riemann: The Birth of the Riemannian Geometry}

Bernhard Riemann, who belongs among the Giants of Mathematics, showed a great interest for defining the properties of Space and Time. Like Carl Gauss, his famous Teacher, Riemann conceived the geometrical properties of Space and Time as a subject of inquiry within the Science of Mechanics, properties which had to be measured after conducting the appropriate experiments.

Riemann develops a novel kind of Geometry, which encompasses all the Non-Euclidean Geometries proposed up to his era, that is the Spherical Geometry, already known and used extensively by the Hellenistic Astronomers and the Bolyai-Lobachevski's Hyperbolic Geometry. The Riemannian Geometry surpasses them even furthermore, by generalizing their structure and logical texture and by exposing clearly and systematically all of their attributes of primal importance (Vizgin, 2011). One of his greatest achievements is the clarification between the notions of infinite and unlimited.

The work of Bernhard Riemann led not only to a clarification of the mathematical notion about an abstract system of Geometry, but also served as a vehicle and an insight for the clarification and determination of Physical Space, as well.

Namely, the Physicists were not obligated to place the framework of their theories within the context of Euclidean Geometry, since they could choose among many variants of Riemannian manifolds and use many versions of metrics, in order to express kinematically and dynamically the structure of their own theoretical proposals.

Up to the age of Riemann, the only existing and available Geometry which served as a prototype about the structure of Space, Time, Motion and the Dynamics of Masses and Charges under the influence of Forces, at first, or under the influences of Fields, at second within the flow of Historical time, was the Euclidean Geometry.

This conclusion was obvious, at the end of the $19^{\text {th }}$ century, only in the eyes of few Physicists, including Riemann, who understood that the determination of the metric structure of the manifold of Space and Time ought to be found only within the context of the forces that act on it and especially the Gravitational forces generated by the Mass distributions within the Universe, as Riemann himself was convinced (Ben-Menahem, 2009).

\subsection{Henry Poincare: The Introduction of Chaos in Celestial Mechanics}

Henry Poincare, one of the greatest Mathematicians and Physicists of all ages, did not only contribute greatly into the Theory of Special Relativity, but also offered a decisive step of the conceptual and functional enrichment within the realm of Classical Mechanics, since he can be considered as the Father for introducing the Science of Chaos into Celestial Mechanics, especially during his engagement with the famous "Three Body Problem" (Ivancevic and Ivancevic, 2007).

Henry Poincare introduces several geometric and topological notions into Celestial Mechanics, such as the notion of the Poincare Map and is one of the first to recognize the structure of the geometrical entity we would call in our epoch as the "strange attractor". The pioneering work of Henry Poincare opened a completely novel view of the kinematical and dynamical processes of gravitationally interacting physical bodies, in the description of our Solar System, in the formation and evolution of clusters of stars and galaxies, of the collision and interaction between galaxies, just to mention a few basic thematic issues which are extensively studied in our epoch (Contopoulos, 2003).

\section{THE UNIVERSE ACCORDING TO ALBERT EINSTEIN}

\subsection{Introduction}

The hypothesis of the existence of the motionless ether, the carrier of the electromagnetic fields, as these are described according to Maxwell's Theory of 
Electromagnetism, seemed to save the assumption of the existence of the Absolute Space and Time, among other theoretical problematic situations of the era between the end of the 19th century and up to Albert Einstein's introduction of the Theory of Special Relativity. The inertial frame of reference of the ether could be identified with the reference frame Newton supposed generations ago, a notion also developed by the majority of the Postnewtonian scientists. It is remarkable to comment that both the classical Theory of Lorentz, as well as Einstein's Theory of Special Relativity, can interpret in a self-consistent way the outcomes of the famous Michelson-Morley experiment, conducted to trace the existence of this very exotic universal substratum, the ether. On the other hand, the existence of ether, according to Lorentz's theory, still remained an unobservable quantity. The problematic issue about the existence of ether, together with all its epistemological and methodological, even metaphysical complications, was going to be proven as one important node in the "historical tree" towards the birth and the evolution of the Theory of Special Relativity.

\subsection{Space, Time and Mass formulated within the Theory of Special Relativity}

We mention briefly some of the main characteristics of Albert Einstein's Theory of Special Relativity, since there exist already a plethora of textbooks and works devoted to the study of one of the most fundamental Physical Theories (Rindler, 1991).

The introduction of Spacetime can be regarded as one of the most fundamental characteristics of the Theory of Special Relativity. As stated by Hermann Minkowski, Space and Time loose their independent existence and form the amalgam of Spacetime, where the motion of the physical bodies take place and in which the primary notions of the physical Event, the Space, the Time and the Inertial mass obtain their operational definition.

The physical Time looses its absolute Newtonian nature and relies heavily on the process of synchronization, as already proposed by Poincare, as well. The structure of Spacetime is regarded to be isotropic and homogeneous, while the kinematical effects on the length of a physical body and on the flow of time, as measured by clocks in the inertial frame of the observer, compared to the same measurements conducted in another frame of reference, obey the Lorentz- Einstein transformations between these two, or between any, Inertial systems of reference. The Inertial mass, as conceived within the Theory of Special Relativity, is velocity-depended and furthermore the Inertial mass can be converted into Energy, a fact that places these two primary physical Notions on equal footing.

Yet, the Spacetime of the Theory of Special Relativity bears some resemblances with the Newtonian concepts, in the sense of the Parmenidean nature of Spacetime, in which all Inertial frames of Reference are endowed. Also, the Theory of Gravity cannot be encompassed within the Theory of Special Relativity.

The introduction of Spacetime can be regarded as one of the most fundamental characteristics of the Theory of Special Relativity. As stated by Hermann Minkowski, Space and Time loose their independent existence and form the amalgam of Spacetime, where the motion of the physical bodies take place and in which the primary notions of the physical Event, the Space, the Time and the Inertial mass obtain their operational definition.

The physical Time looses its absolute Newtonian nature and relies heavily on the process of synchronization, as already proposed by Poincare, as well. The structure of Spacetime is regarded to be isotropic and homogeneous, while the kinematical effects on the length of a physical body and on the flow of time, as measured by clocks in the inertial frame of the observer, compared to the same measurements conducted in another frame of reference, obey the Lorentz- Einstein transformations between these two, or between any, Inertial systems of reference. The Inertial mass, as conceived within the Theory of Special Relativity, is velocity-depended and furthermore the Inertial mass can be converted into Energy, a fact that places these two primary physical Notions on equal footing.

Yet, the Spacetime of the Theory of Special Relativity bears some resemblances with the Newtonian concepts, in the sense of the Parmenidean nature of Spacetime, in which all Inertial frames of Reference are endowed. Also, the Theory of Gravity cannot be encompassed within the Theory of Special Relativity.

\subsection{The Advent of the Theory of General Relativity}

The formulation of the Theory of General Relativity, as being introduced by Albert Einstein and further developed by a long chain of successive contributions of the greatest minds that followed, is also a topic which has been extensively studied and which is still under active development. Therefore, we shall mention briefly some characteristics of this rich conceptual structure, which can be regarded as fundamental. 
The theoretical success of the Theory of Special Relativity, especially the inclusion of Maxwell's Theory of Electromagnetism into the fundamental Laws of Physics which are valid in any Inertial Frame of Reference, its simplicity and its aesthetic beauty, was tremendous. On the other hand, the Theory of Special Relativity does not include within its framework the conceptual and mathematical description of the other, then known, fundamental interaction, namely the formulation of a relativistic Theory of Gravity was urgently needed.

Within the framework of the Theory of General Relativity we encounter the Principle of Equivalence, the Principle of General Relativity, the Principle of General Covariance and Mach's Principle, of the relativization of Inertia.

The Principle of Equivalence plays a fundamental role, at least in the first introduction of the Theory of General Relativity by Albert Einstein. Up to our times, there exist various formulations of the Theory of General Relativity, where the Principle of Equivalence can also be regarded as a theorem within the overall framework of the theory. The Principle of Equivalence, as stated by Einstein, leads us to the issue of establishing the relativistic laws obeyed by the inertial-gravitational field. Within the understanding of the Theory of General Relativity, the mass acts on spacetime and thus gets related with its curvature, while spacetime acts on the mass and determines the trajectories of its motion. The insights of Leibniz and the vision of Riemann contribute greatly in order to establish the "universal theatrical scene", where the gravitational and the electromagnetic Fields act upon moving bodies and in turn are acted upon and where spacetime is considered to be a Riemannian manifold, to which the relevant metric is attributed.

Also, within the formulation of the Theory of General Relativity, the problematic issue of the three-fold nature of Mass, that is the Inertial mass, the Passive gravitational mass and the Active gravitational mass, finds its epistemological solution, since these three different concepts are regarded to be identical.

With the statement of the field equations of the Theory of General Relativity, the Universe is geometrized and its Geometrical structure becomes a problem for the Science of Physics (Misner et al., 1973).

We have to stress the fact that the Theory of Newtonian Gravitation can also be stated within the geometrical language Einstein's Theory of General Relativity is formulated and stated. Thus, the crucial points, in which these two approaches differ, can be exposed in a direct way.
It is an astonishing fact of the History of Physics and of Modern Philosophy, as well, that the irregularity of Uranus' orbit finally came to the discovery of the new planet Neptune, while the discrepancies in Mercury's orbit led to one of the triumphs of the Theory of General Relativity.

\subsection{The Cosmological Work of Alexander Friedmann}

Alexander Friedmann is one of the most prominent Figures within the History of Gravity, one Physicists who shapes the foundations of modern Cosmology (Belenkyi, 2012). Alexander Friedmann proposes as a true pioneer a totally novel solution for the field equations developed by Einstein, "entering to waters no one yet has crossed".

Alexander Friedmann demonstrates for the first time non-static solutions for the field equations of the Theory of General Relativity and offers many variants for the cosmological evolution of the Universe. His solutions regard a perfect-fluid Cosmos of uniform density, which evolves dynamically, surpassing the Parmidean notion of the Universe, a stable, static, homogenous and isotropic Cosmos, which Einstein himself considered as primal, during the first phases of the introduction of the theory. Alexander Friedmann is nowadays considered to be as one of the prominent Figures in the History of Gravity, which naturally encloses the Modern Cosmology, as well.

\section{CONCLUSION}

The fundamental interactions among the physical bodies and especially the gravitational interaction, the interaction whose discovery appears as first in the catalogue of the four fundamental interactions, with the second known interaction, in the historical flow of time, the electromagnetic interaction, which served as a fountain for the Theory of Special Relativity, through their intense study served as the motor mechanisms for the development of the Science of Physics and more generally of all branches of Technology, Philosophy, Theology and all spaces of social discourse, such as the Arts and the Social phenomena, as well as the everyday life of the layman, in each historical epoch and for every great Civilization.

The History of Gravity offers us an excellent account about the formation of primary concepts within the History of Physics and shows the complicated path of interacting Paradigms within the Science of Physics, 
considered in their historical succession of appearance, as rivals, or as completing each other and determines our deeper understanding about these fundamental concepts at the begin of the 21 st century. All these notions attain their true functional and operational meaning within the framework of each School and of each Period within the development of Physics. Often, all the important personalities contributing to the creation of a Theory of Gravity use the "old" material in order to built their own novel worlds and Paradigms.

In each Paradigm of the Gravitational interaction, as this transforms and evolves through History by the successive contributions of Scholars and Scientists and also at the transition of the old to the new Paradigm, the fundamental notions of Space, of Time, of Motion, of Inertia, of Mass and of Field, are being continuously redefined and alter their status within the conceptual, logical, methodological and experimental structure of the theory. In each of these Paradigms, World-views, philosophical and physical Principles meet the quantitatively testable propositions of the theory. Each prevailing Paradigm, the Atomistic, the Aristotelian, the Neoplatonic, the Keplerian, the Newtonian, the Machian or the Einsteinian, carries with itself a host of conceptual problematic issues and inherent epistemological defects, as well as fine details of the Theory which have to be solved.

Thus, the History of Gravity is of importance, both for the Historian, the Philosopher of Science, as well as for the working Theorist in the scientific discipline of the Theory of Gravity, as well as in other related disciplines.

\section{ACKNOWLEDGEMENT}

I gratefully acknowledge Pr. Xenophon Moussas, Pr. Efstratios Theodossiou and Mr. Emmanuel Manoussos for critical readings and many interesting discussions.

\section{REFERENCES}

Arnold, V.I., 1989. Mathematical Methods of Classical Mechanics. 1st Edn., Springer, New York, ISBN-10: 0387968903, pp: 508.

Belenkyi, A., 2012. Alexander Friedmann and the origins of modern cosmology. Phys. Today, 65: 38: 38-43. DOI: 10.1063/PT.3.1750

Ben-Menahem, A., 2009. Demise of the Dogmatic Universe. In: Historical Encyclopedia of Natural and Mathematical Sciences, Springer, Berlin, ISBN-10: 3540688315, pp: 2801-5079.
Boschiero, L., 2010. The presocratic origins of modern science. Metascience, 19: 465-467. DOI: 10.1007/s11016-010-9362-y

Bouquiaux, L., 2008. Leibniz Against the Unreasonable Newtonian Physics. In: Leibniz: What Kind of Rationalist? What Kind of Rationalist? Dascal, M., (Ed.), Springer, Dordrecht, ISBN-10: 1402086687, pp: 99-110.

Burnet, J., 1914. Greek Philosophy. Part 1. Thales to Plato. 1st Edn., London, Macmillan and Co., pp: 360.

Contopoulos, G., 2003. Order and Chaos in Astronomy. In: Galaxies and Chaos, Contopoulos, G., Voglis, N., (Eds.), Springer, Berlin, ISBN-10: 3540404708, pp: 3-29.

Cornford, F.M., 1922. Mysticism and science in the pythagorean tradition. Classical Q., 16: 137-150. DOI: $10.1017 / \mathrm{S} 0009838800002718$

Dicke, R.H., 2011. Cosmology, Mach's principle and relativity. Resonance, 16: 372-391. DOI: 10.1007/s12045-011-0043-y

Dreyer, J.L.E., 2011. A history of Astronomy from Thales to Kepler. 1st Edn., Dover Publications, ISBN-10: 0486600793, pp: 464.

Ducheyne, S., 2007. The General Scholium: Some Notes on Newton's Published and Unpublished Endeavours.

Elazar, M., 2011. Honore fabri and the concept of impetus: A bridge between paradigms. Boston Stud. Philosophy Sci. DOI: 10.1007/978-94-007-1605-6

Ferraro, R., 2007. Inertia and Gravity. In: Einstein's Space-Time: An Introduction to Special and General Relativity, Ferraro, R. (Ed.), Springer, New York, ISBN-10: 0387699465.

Henriksson, G., 2009. Ten solar eclipses show that the Antikythera mechanism was constructed for use on Sicily. Proceeding of the 17th Annual Meeting on European Society for Astronomy in Culture (ESAC' 09), Alexandria Library, Alexandria, Egypt.

Ivancevic, V. and G. Ivancevic, 2007. Introduction to Attractors and Chaos. In: High-Dimensional Chaotic and Attractor Systems, Ivancevic, V. and G. Ivancevic (Eds.), Springer, Netherlands, ISBN: 9781402054556, pp: 1-151.

Jacob, A., 1991. The Neoplatonic Conception of Nature in More, Cudworth and Berkeley. In: The Uses of Antiquity, Gaukroger, S. (Ed.), Springer, Netherlands, pp: 101-121. 
Jammer, M., 1954. Concepts of Space: The History of Theories of Space in Physics. 1st Edn., Harvard University Press, Cambridge (Mass).

Jammer, M., 1957. Concepts of Force: A Study in the Foundations of Dynamics. 1st Edn., Harvard University Press, Cambridge (Mass).

Jammer, M., 1997. Concepts of Mass in Classical and Modern Physics. 1st Edn., Courier Dover Publications, Mineola, New York, ISBN-10: 0486299988, pp: 230.

Kalachanis, K., 2011. On example and image in the work of John industriousness. Paradigm and the Icon, in the work of John Philoponus. PhD Dissertation, University of Athens, Athens, Greece (in Greek).

Kuhn, T.S., 1996. The Structure of Scientific Revolutions. 3rd Edn., The University of Chicago Press, Chicago, ISBN-10: 0226458083, pp: 226.

Misner, C.W., K.S. Thorne and J.A. Wheeler, 1973. Gravitation. 1st Ed., W. H. Freeman, New York, ISBN-10: 0716703440, pp: 1279.

Moussas, X., 2012. The Antikythera Mechanism: Table, The First Mechanical Universe. Hellenic Physical, Greek.

Moussas, X., J. Seiradakis, T. Freeth, M. Edmunds and Y. Bitsakis et al., 2007. Communicating astronomy with the public.

Narlikar, J.V., 2011. Mach's principle. Resonance, 16: 310-321. DOI: 10.1007/s12045-011-0037-9

Nowacki, H., 2010. The Heritage of Archimedes in Ship Hydrostatics: 2000 Years to Applications. In: The Genius of Archimedes -- 23 Centuries of Influence on Mathematics, Science and Engineering, Paipetis, S.A. and M. Ceccarelli (Eds.), Springer, Dordrecht, Netherlands, ISBN-10: 9048190916, pp: 227-249.
Papaspirou, P., 2012. Johannes Kepler: His place in Astronomy. Proc. Sci.

Rindler, W., 1991. Introduction to Special Relativity. 1st Edn., Oxford University Press, Oxford, ISBN-10: 0198539525, pp: 169.

Russo, L. and S. Levy, 2004. The Forgotten Revolution: How Science was Born in $300 \mathrm{BC}$ and Why it Had to be Reborn. 1st Edn., Springer, Berlin, ISBN-10: 3540203966, pp: 487.

Spandagos, E., 2011. Life and work of archimedes. Aethra, Greek.

Suisky, D., 2008. Euler's Program for Mechanics. In: Euler as Physicist, Suisky, D. (Ed.), Springer, Berlin Heidelberg, ISBN-10: 3540748652, pp: 101-194.

Theodossiou, E., E. Danezis, V.N. Manimanis and E.M. Kalyva, 2002. From Pythagoreans to Kepler: The dispute between the geocentric and the heliocentric systems. J. Astronomical History Heritage, 5: 89-98.

Vizgin, V.P., 2011. The General Theory of Relativity: The Core of the Program of Unified Field Theories. In: Unified Field Theories: In the First Third of the Twentieth Century, Vizgin, V.P. (Ed.), Springer, Basel AG., ISBN-10: 3034801742, pp: 47-70.

Westfall, R.S., 1973. Newton and the fudge factor. Science, 179: 751-758. DOI: 10.1126/science. 179.4075 .751

Wilson, C.A., 1970. From Kepler's laws, so-called, to universal gravitation: Empirical factors. Arch. History Exact Sci., 6: 89-170. DOI: 10.1007/BF00328337 